\title{
Redundant space calibration of hexagonal and Y-shaped beamforming radars and interferometric radiometers
}

\author{
A. CAMPS*, F. TORRES \\ Department of Signal Theory and Communications, Universitat Politècnica de \\ Catalunya, Campus Nord D3, E-08034 Barcelona, Spain \\ P. LOPEZ-DEKKER and S. J. FRASIER \\ Department of Electrical and Computer Engineering, University of \\ Massachusetts, Amherst, MA 01003, USA
}

(Received 19 December 2000; in final form 14 October 2002)

\begin{abstract}
The calibration of large antenna arrays in the absence of a beamforming point source is a common problem in beamforming radars and interferometric radiometers. In this paper the fundamentals of the redundant space calibration (RSC) method for phase and amplitude are reviewed, pointing out the parallelism between the active and passive cases, the technique is then applied in a general and systematic way to two cases of interest: hexagonal planar and Y-shaped arrays, which are known to be the optimum periodic twodimensional configurations. In both cases, the system of equations is determined taking into account the available redundancies and symmetries of these structures. The performance of the RSC method is analysed in terms of the propagation of errors in reference phase/amplitude. The technique is then considered for two hexagonal array systems: the Turbulent Eddy Profiler (TEP), a volume-imaging radar of the lower atmosphere developed at the University of Massachusetts, and the Microwave Imaging Radiometer by Aperture Synthesis (MIRAS), an L-band interferometric radiometer to be launched in 2005 in the Earth Explorer Opportunity Mission-Soil Moisture and Ocean Salinity Mission (SMOS) of the European Space Agency (ESA). In the TEP case the proposed scheme stabilizes an otherwise ill-posed inversion problem, and in the MIRAS case it presents an alternative calibration method to the noise-injection one, without any additional hardware required.
\end{abstract}

\section{Introduction}

In a radar system, echoes from distributed scattering sources much smaller than the resolution cell, such as sea waves, vegetation or the atmosphere, are generally incoherent. Attia and Steinberg (1989) showed that the spatial cross-correlation $V_{k l}$ computed between the echoes $b_{k, J}(t)=S_{k, l}(t) \exp \left(j \varphi_{k, l}(t)\right)$ collected by two different elements $k$ and $l$ in the array, at a certain range gate, is a sample of the Fourier transform of the intensity distribution $I(\xi, \eta)$ evaluated at the spatial frequency $\left(u_{k l}\right.$,

*Corresponding author; e-mail: camps@tsc.upc.es 
$v_{k l}$ ) determined by the antenna separation normalized to the wavelength (baseline $k-l)$ :

$$
\begin{aligned}
V_{k l} \hat{=} V\left(u_{k l}, v_{k l}\right)=1 / 2\left\langle b_{k}(t) b_{l}^{*}(t)\right\rangle & =\iint_{\xi^{2}+\eta^{2} \leqslant 1} I(\xi, \eta) \exp \left(-j 2 \pi\left(u_{k l} \xi+v_{k l} \eta\right)\right) d \xi d \eta \\
\left(u_{k l}, v_{k l}\right) & =\left(x_{l}-x_{k}, y_{l}-y_{k}\right) / \lambda
\end{aligned}
$$

where $\xi=\sin \theta \cos \varphi$ and $\eta=\sin \theta \sin \varphi$ are the directing $\operatorname{cosines}$ with respect to the $x$ and $y$ axes. Equation(1) is a version of the Van Cittert-Zernike theorem (Born and Wolf 1975). Equation(1) also holds in the passive case, but the signals $b_{k, l}(t)=S_{k, l}(t) \exp \left(j \varphi_{k, l}(t)\right)$ are the electric fields (thermal noise) received at the elements' position (Thompson et al. 1986).

In the active case (radar) the intensity distribution $I(\xi, \eta)$ is created by the backscattered power as illuminated by the pattern of the transmitting and receiving antennas. In the passive case, the intensity distribution is the brightness temperature distribution multiplied by the antenna pattern of the receiving antennas, the obliquity factor $1 / \sqrt{1-\xi^{2}-\eta^{2}}=1 / \cos \theta$ and the fringe-washing function that accounts for spatial decorrelation effects.

When the array elements have uncorrelated phase errors among them, $f_{k}$ and $f_{l}$, the measured phase $\phi_{k l}^{\mathrm{raw}}$ of the measured complex cross-correlation $V_{k l}^{\mathrm{raw}}$ is given by

$$
\phi_{k l}^{\mathrm{raw}}=f_{k}-f_{l}+\phi_{k l}
$$

where $\phi_{k l}$ is the phase of $V_{k l}$, measured by an ideal system (Hamaker et al. 1977).

In a similar way, the amplitude of the measured cross-correlation is given by

$$
\left|V_{k l}^{\mathrm{raw}}\right|=g_{k} g_{l}\left|V_{k l}\right|
$$

where $g_{k}$ and $g_{l}$ are the separable instrumental amplitude factors of the elements $k$ and $l$ in the array, and $\left|V_{k l}\right|$ is the amplitude of the complex cross-correlation that would be measured by an ideal instrument. Equation(3) can be linearized by taking logarithms

$$
\ln \left(\left|V_{k l}^{\mathrm{raw}}\right|\right)=\ln \left(g_{k}\right)+\ln \left(g_{l}\right)+\ln \left(\left|V_{k l}\right|\right)
$$

or, in a simpler form

$$
A_{k l}^{\mathrm{raw}}=G_{k}+G_{l}+A_{k l}
$$

with the obvious definitions $G_{k, l}=\ln \left(g_{k, l}\right), A_{k l}=\ln \left(\left|V_{k l}\right|\right)$ and $A_{k l}^{\text {raw }}=\ln \left(\left|V_{k l}^{\text {raw }}\right|\right)$.

Since the spatial cross-correlation is only a function of the antenna separation, all the antennas spaced the same distance in the same direction measure the same baseline $\left(u_{k l}, v_{k l}\right)$, which are redundant baselines. The phase and amplitude of the available redundant baselines (equations (2) and (4)) allows to define a system of equations ( $\$ 2.1$ and 3.1) that can be solved for the unknown phase/amplitude instrumental errors $\left(f_{k, l}\right.$ and $\left.g_{k, l}\right)$. This is the concept of the redundant space calibration (RSC). Since in most cases the scene imaged has slow spatial variations which are tapered by the antenna pattern itself, $\left|V\left(u_{k l}, v_{k l}\right)\right|$ decreases with increasing antenna spacing (larger $\left|\left(u_{k l}, v_{k l}\right)\right|$ ), and only the shortest baselines are used, since they have the highest signal-to-noise ratio (SNR) and provide the best phase/amplitude estimates.

This paper is divided into four sections. In $\S 2$ and $\S 3$, the RSC is applied to hexagonal planar and to $\mathrm{Y}$-shaped arrays, respectively. In both cases, the number 
of equations, unknowns and system's rank are studied first. Then, the propagation of deterministic errors in the phase references is studied. Finally, in $\S 4$, these results are applied to predict the performance of two particular instruments: the Turbulent Eddy Profiler (TEP) and the Microwave Imaging Radiometer by Aperture Synthesis (MIRAS).

\section{RSC in hexagonal planar arrays}

Figure 1(a) shows a hexagonal planar array with 91 elements, as in TEP (Mead et al. 1998), although the results are general and can be applied to arrays with an arbitrary number of elements. Without loss of generality the phase of the central element is set to zero $\left(f_{0}=0\right)$, since a constant phase term added to all the elements is irrelevant. Also, the synthetic beam shape is not altered if the elements' amplitudes are referred to that of element $0\left(g_{0}=1\right.$, or $\left.G_{0}=0\right)$.

\subsection{Available redundancies and equations}

Figure 1 $(a)$ shows the minimum number of redundant baselines required for solving the system of equations for all $f_{k}$ as shown hereafter. A set of RSC equations can be defined along each of the linear arrays (vertical lines marked in figure $1(a)$ ). For simplicity, we will begin our discussion with the array formed by the first ring (antennas 0, 1, 2, 3, 4, 5 and 6 in figure 1(a)). According to equation (2) the following relationships can be written for the phase errors

$$
\phi_{56}^{\text {raw }}=f_{5}-f_{6}+\phi_{01}, \phi_{01}^{\text {raw }}=0-f_{1}+\phi_{01}, \phi_{40}^{\text {raw }}=f_{4}-0+\phi_{01}, \phi_{32}^{\text {raw }}=f_{3}-f_{2}+\phi_{01}
$$

In equation (5) there are seven unknowns $\left(\phi_{01}, f_{1} \ldots f_{6}\right)$ and four equations. Since all the phases must be related to one $\left(f_{0}=0\right)$, a set of diagonal redundant baselines is required to link them all. To do so, at least two new equations are needed, for

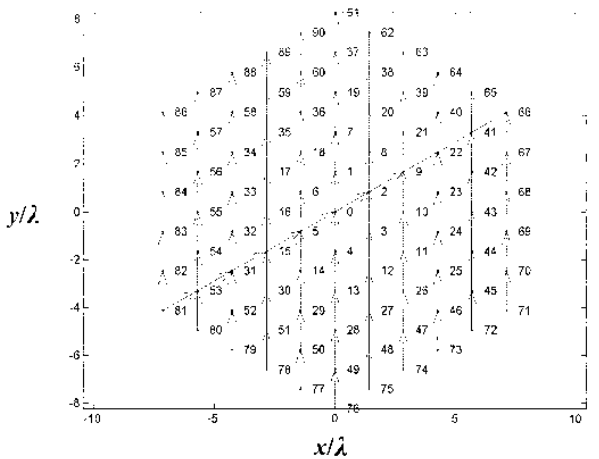

$(a)$

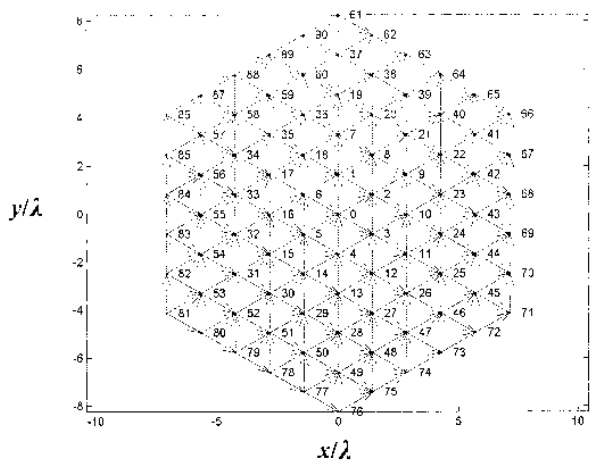

(b)

Figure 1. Baselines used to define the RSC for $(a)$ case 1 and $(b)$ case 2 in a hexagonal planar array. Each arrow indicates a baseline. The 91 antennas grouped into five rings: first ring, elements 1-6; second ring, elements 7-18; third ring, elements 19-36; fourth ring, elements 37-60; fifth ring, elements 61-90. Shortest redundant baselines: (a) 01 and 02 , and $(b) 01,02$ and 03 . 
example (figure 1(a)):

$$
\phi_{02}^{\mathrm{raw}}=0-f_{2}+\phi_{02}, \phi_{50}^{\mathrm{raw}}=f_{5}-0+\phi_{02}
$$

which just introduce a new unknown $\phi_{02}$.

Following a similar procedure, according to equation(4) the following relationships can be written for the amplitude factors

$A_{56}^{\mathrm{raw}}=G_{5}+G_{6}+A_{01}, A_{01}^{\mathrm{raw}}=0+G_{1}+A_{01}, A_{40}^{\mathrm{raw}}=G_{4}+0+A_{01}, A_{32}^{\mathrm{raw}}=G_{3}+G_{2}+A_{01}$

and also

$$
A_{02}^{\mathrm{raw}}=0+G_{2}+A_{02}, A_{50}^{\mathrm{raw}}=G_{5}+0+A_{02}
$$

In the general case of an array with $n_{r}$ rings ( $n_{r}=5$ rings in figure 1$)$ two systems of equations can be written

$$
\bar{\phi}^{\mathrm{raw}}=\bar{M}\left[\begin{array}{c}
\bar{f} \\
\phi_{01} \\
\phi_{02}
\end{array}\right] \quad \bar{A}^{\mathrm{raw}}=\bar{N}\left[\begin{array}{c}
\bar{G} \\
A_{01} \\
A_{02}
\end{array}\right]
$$

These systems of equations contain the strict minimum number of equations and will be referred to as case 1 . Their properties are summarized in table 1 . In both cases, there are two more unknowns than equations. Hence, two reference element phases/amplitudes (e.g. $f_{1}$ and $f_{2}$, or $G_{1}$ and $G_{2}$ ), or phases/amplitudes of the complex cross-correlations (e.g. $\phi_{01}$ and $\phi_{02}$, or $A_{01}$ and $A_{02}$ ) have to be determined. These two phase unknowns correspond to a pointing uncertainty of the focused beam (Attia and Steinberg 1989).

Figure 1 $(b)$ shows all the shortest baselines available in a hexagonal planar array. They are all parallel to the baselines $0-1,0-2$ and $0-3$. The corresponding system of equations will be referred to as case 2 . Although, strictly speaking, more

\begin{tabular}{|c|c|c|c|c|}
\hline & $\begin{array}{l}\text { Number of } \\
\text { equations }\end{array}$ & $\begin{array}{l}\text { Number of } \\
\text { unknowns }\end{array}$ & $\begin{array}{l}\text { Rank of the } \\
\text { system of } \\
\text { equations }\end{array}$ & Comments \\
\hline \multicolumn{5}{|l|}{ Phase RSC } \\
\hline $\begin{array}{l}\text { Case } 1 \text { : minimum } \\
\text { number of equations }\end{array}$ & $3 n_{r}^{2}+3 n_{r}$ & $3 n_{r}^{2}+3 n_{r}+2$ & $3 n_{r}^{2}+3 n_{r}$ & \multirow{2}{*}{$\begin{array}{l}\text { Two reference phases to } \\
\text { be determined: } f_{1} \text { and } f_{2} \\
\text { or } \phi_{01} \text { and } \phi_{02}{ }^{*} \\
\text { Two reference phases to } \\
\text { be determined: } f_{1} \text { and } f_{2} \\
\text { or } \phi_{01} \text { and } \phi_{02}{ }^{*}\end{array}$} \\
\hline $\begin{array}{l}\text { Case 2: maximum } \\
\text { number of equations }\end{array}$ & $9 n_{r}^{2}+3 n_{r}$ & $3 n_{r}^{2}+3 n_{r} 3$ & $3 n_{r}^{2}+3 n_{r}+1$ & \\
\hline $\begin{array}{l}\text { Amplitude RSC } \\
\text { Case 1: minimum }\end{array}$ & $3 n^{2}+3 n_{r}$ & $3 n^{2}+3 n_{r}+2$ & $3 n^{2}+3 n_{r}$ & \multirow{3}{*}{$\begin{array}{l}\text { Two reference amplitudes } \\
\text { to be determined: } G_{1} \text { and } \\
G_{2} \text { or } A_{01} \text { and } A_{02} \\
\text { Determined system } \dagger\end{array}$} \\
\hline number of equations & & & & \\
\hline $\begin{array}{l}\text { Case 2: maximum } \\
\text { number of equations }\end{array}$ & $9 n_{r}^{2}+3 n_{r}$ & $3 n_{r}^{2}+3 n_{r}+3$ & $3 n_{r}^{2}+3 n_{r}+3$ & \\
\hline
\end{tabular}

Table 1. Hexagonal planar array: system of equations properties for phase/amplitude redundant space calibration (RSC).

*Correspond to an azimuth an elevation pointing error of the beam.

†Absolute calibration requires knowledge of $g_{0}\left(g_{0}=1, G_{0}=0\right)$. 
equations can be defined, it can be shown that all of them are linear combinations of the ones presented in figure $1(b)$. Their properties are summarized in table 1 . As for case 1, there are always two more phase unknowns than linear independent equations. However, for amplitude RSC, the case 1 system of equations requires the determination of two amplitude terms, e.g. $G_{1}$ and $G_{2}$, while the case 2 system provides all the cross-correlations, and amplitude terms. Once the reference phases and amplitudes are determined, equation (9) is solved by direct inversion (case 1, same number of unknowns than equations) or by least squares (case 2, more equations than unknowns).

\subsection{Error propagation in the reference phases/amplitudes}

In $\$ 2.1$, it was shown that two reference phases are required $\left(f_{1}\right.$ and $f_{2}$ or $\phi_{01}$ and $\left.\phi_{02}\right)$. An error in the estimation of $f_{1}$ or $f_{2}$ is equivalent to an error in the estimation of $\phi_{01}$ or $\phi_{02}$ respectively, since $f_{0}=0$ by convention. In the radar case, the transmitting antenna beam defines the illumination of the scene, which is usually symmetric and $\phi_{01}=\phi_{02}=0^{\circ}$. If there is a pointing error $\left(\theta_{0}, \varphi_{0}\right)$, a phase error $\Delta \phi_{k l}=-2 \pi\left(u_{k l} \sin \theta_{0} \cos \varphi_{0}+v_{k l} \sin \theta_{0} \sin \varphi_{0}\right)$ is induced, which forces the focused beam to point towards the direction of maximum intensity (Attia and Steinberg 1989). Alternatively, $f_{1}$ and $f_{2}$ can be determined from the cross-correlations formed by elements 0 and 1 (baseline $0-1$ ) and 0 and 2 (baseline $0-2$ ) when a CW (continuous wave) tone is injected to elements 0, 1 and 2 (Mead et al. 1998). In an interferometric radiometer, $\phi_{01}$ and $\phi_{02}$ depend on the brightness temperature distribution being imaged and cannot be estimated. Therefore, $f_{1}$ and $f_{2}$ have to be determined by other means, such as the injection of correlated noise (Torres et al. 1996).

Figure 2 shows the propagation of an error in the reference phases $f_{1}$ (or $\phi_{01}$ ) and $f_{2}$ (or $\phi_{02}$ ) in the estimated phase of the other elements. Results computed using

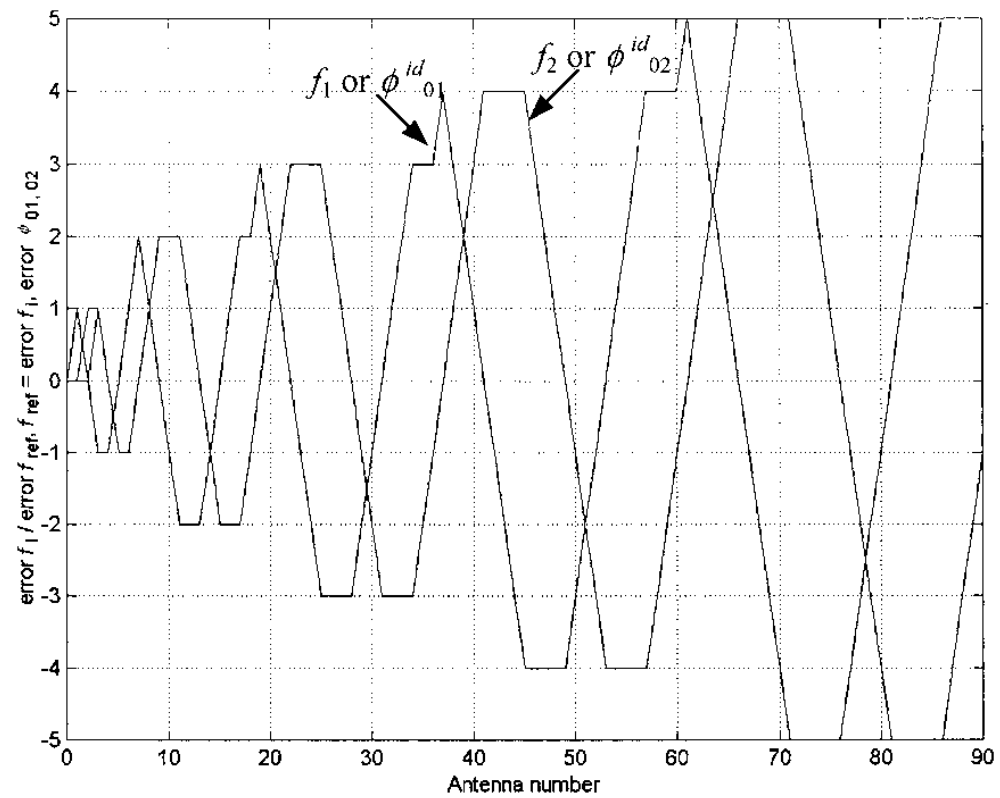

Figure 2. Amplification of reference phase errors in the estimation of elements phase. 
equation (9) for cases 1 and 2 show the same behaviour. Note that an error in $f_{1}$ (or $\left.\phi_{01}\right)$ does not propagate along the direction of the baselines $0-2$ and $0-5$. In addition, the error amplification factor has maximum amplitude (equal to the ring number: 1 for elements $1-6,2$ for elements 7-18, etc; figure $1(a)$ ) in all the elements comprised in the sector defined by the baselines $0-1$ and $0-6$, and the sector defined by the baselines $0-3$ and $0-4$. Similarly, an error in $f_{2}$ (or $\phi_{02}$ ) does not propagate along the direction of the baselines $0-1$ and $0-4$, and the error amplification factor is maximum in all the elements comprised in the sector defined by the baselines $0-2$ and $0-3$, and the sector defined by the baselines $0-1$ and $0-6$.

\section{RSC in Y-shaped arrays}

Figure 3(a) shows a Y-shaped array with 23 elements per arm, plus one in the centre, and three additional central elements for calibration purposes (Martin-Neira and Goutoule 1997, Silvestrin et al. 2001). Figure 3(b) shows a detail of the central part indicating all the available cross-correlations in this structure. The three extra elements (labelled $3 n_{e l}+1,3 n_{e l}+2$ and $3 n_{e l}+3$ ) establish inter-arms redundant baselines. As with hexagonal planar arrays, without loss of generality, the phase of the centre element can be set to zero $\left(f_{0}=0\right)$ and the elements amplitude can be referred to it $\left(g_{0}=1, G_{0}=1\right)$.

\subsection{Available redundancies and number of equations}

In a Y-array with $n_{e l}$ elements per arm $\left(n_{e l}=23\right.$ in figure $\left.3(a)\right)$, the set of RSC equations can only be defined along each arm of the $\mathrm{Y}$ structure. This means that, including the central element (numbered as 0 , figures $3(a)$ and $3(b)$ ) in each arm there are $n_{e l}+1$ elements, $n_{e l}$ short baselines (equations) and $n_{e l}+1$ unknowns: one for each element, except the central one, plus one corresponding to the phase/ amplitude of the cross-correlations between pairs $0-1,0-\left(n_{e l}+1\right)$ or $0-\left(2 n_{e l}+1\right)$. Consequently, the cross-correlations in a Y-array (without the three extra elements) lead to a system of equations with $3 n_{e l}$ equations and $3 n_{e l}+3$ unknowns. The

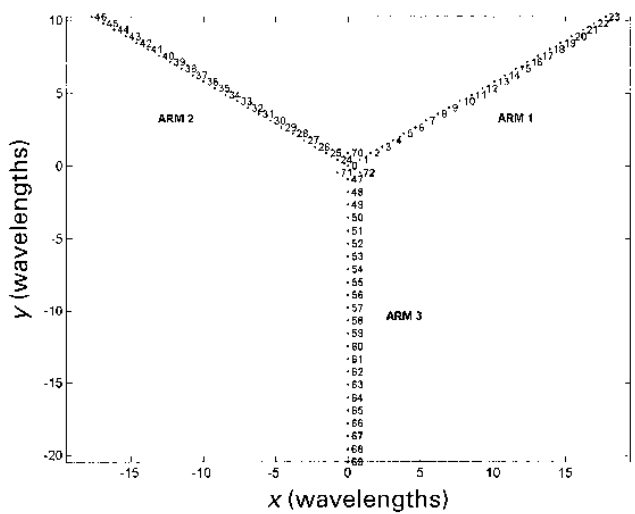

(a)

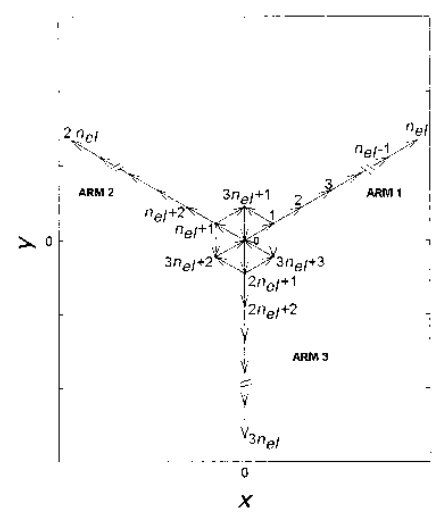

(b)

Figure 3. (a) Structure of a Y-shaped array with 23 antennas per arm spaced $d=0.89$ wavelengths. Extra antennas numbered 70,71 and 72 for phase tracking between arms. (b) Arrows: available complex cross-correlations available in a $\mathrm{Y}$-array with $n_{e l}$ antennas per arm and three additional central antennas $\left(3 n_{e l}+1,3 n_{e l}+2\right.$ and $\left.3 n_{e l}+3\right)$. Arbitrary units. 
Table 2. Y-array: properties of the system of equations for phase/amplitude RSC.

\begin{tabular}{|c|c|c|c|c|}
\hline & $\begin{array}{l}\text { Number of } \\
\text { equations }\end{array}$ & $\begin{array}{l}\text { Number of } \\
\text { unknowns }\end{array}$ & $\begin{array}{l}\text { Rank of the } \\
\text { system of } \\
\text { equations }\end{array}$ & Comments \\
\hline \multicolumn{5}{|l|}{ Phase RSC } \\
\hline Three extra elements & $3 n_{e l}+9$ & $3 n_{e l}+3$ & $3 n_{e l}+4$ & $\begin{array}{l}\text { Two reference phases to } \\
\text { be determined: } f_{1} \text { and } f_{n e l+1} \\
\text { or } \phi_{01} \text { and } \phi_{0, n e l+1}{ }^{*} \\
\text { Two reference phases to } \\
\text { be determined: } f_{1} \text { and } f_{n e l+1} \\
\text { or } \phi_{01} \text { and } \phi_{0, n e l+1}{ }^{*}\end{array}$ \\
\hline Amplitude RSC & & & & \\
\hline No extra element & $3 n_{e l}$ & $3 n_{e l}+3$ & $3 n_{e l}$ & $\begin{array}{l}\text { Two reference amplitudes to } \\
\text { be determined: } G_{1} \text { and } G_{n e l+1} \\
\text { or } A_{01} \text { and } A_{0} \text { nel+1 }\end{array}$ \\
\hline Three extra elements & $3 n_{e l}+9$ & $3 n_{e l}+6$ & $3 n_{e l}+6$ & Determined system $t$ \\
\hline
\end{tabular}

*Correspond to an azimuth an elevation pointing error of the beam.

†Absolute calibration requires knowledge of $g_{0}\left(g_{0}=1, G_{0}=0\right)$.

general expression of the equations for the phase RSC is

$$
\phi_{k-1, k}^{\text {raw }}=f_{k-1}-f_{k}+\phi_{k-1, k}=f_{k-1}-f_{k}+\phi_{0, m}
$$

and for the amplitude RSC

$$
A_{k-1, k}^{\mathrm{raw}}=G_{k-1}+G_{k}+A_{k, k-1}=G_{k-1}+G_{k}+A_{0, m}
$$

with $m=1$ for $k: 1,2 \ldots n_{e l}(\operatorname{arm} 1) ; m=n_{e l}+1$ for $k: n_{e l}+1, n_{e l}+2, \ldots 2 n_{e l}(\operatorname{arm} 2)$ and $m=2 n_{e l}+1$ for $k: 2 n_{e l}+1,2 n_{e l}+2, \ldots 3 n_{e l}$ (arm 3). These equations can be cast in a matricial form as for the hexagonal planar array (equation(9)). The extra central elements link the phase/amplitude unknowns among arms, introducing just a new unknown per element (phase or amplitude), without introducing a new baseline (e.g. the new baseline $1-\left(3 n_{e l}+1\right)$ is redundant with respect to the $\left.0-\left(n_{e l}+1\right)\right)$. Table 2 summarizes the main properties of the systems of equations given by equations (10) and (11), showing the effect of introducing the additional central elements. Without additional extra elements, there are three equations fewer than unknowns (phases and amplitudes). With three redundant elements two reference phases and a reference amplitude need to be determined ${ }^{1}$.

\subsection{Propagation of errors in the reference phases}

Since the system of equations for phase RSC is underdetermined (table 2), the application of the method to Y-arrays also requires two reference phases and the same considerations of hexagonal planar arrays apply. In the solution of equation (9) an error in the reference phases $\left(f_{1}\right.$ or $\phi_{01}$, and $f_{2}$ or $\left.\phi_{02}\right)$ propagates to other elements' phase estimate, increasing with the distance to the centre of the array (figure 4(a)). Propagation of amplitude errors is more important due to the exponential term $g_{k}=\exp \left(G_{k}\right)$ (figure $4(b)$ ).

$\mathrm{Y}$-arrays are so thinned that they are usually operated in interferometric mode, and the system behaves as a synthetic aperture with an element at the position of

\footnotetext{
${ }^{1}$ The third extra element is redundant with the other two in case of failure.
} 


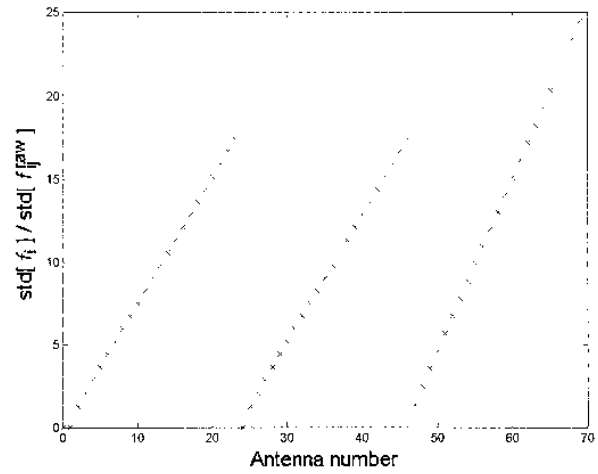

(a)

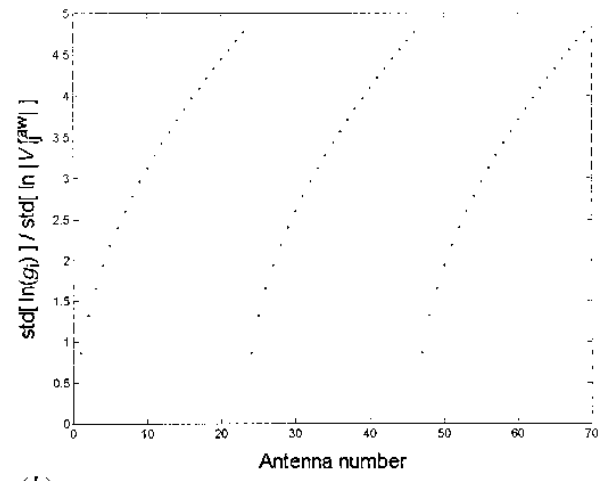

(b)

Figure 4. Amplification of rms (a) phase and $(b)$ amplitude errors along the Y-arms.

each $\left(u_{k l}, v_{k l}\right)$ point (Bara et al. 1998). An interferometric array is more sensitive to phase errors and their propagation than a filled array, since the phase of the measurement $V_{k l}$ is obtained from the difference between the phases of elements $k$ and $l$, which are both affected by the propagation of errors in the estimation of the reference phases $\Delta f_{1}$ and $\Delta f_{n e l+1}$

$$
\Delta \phi_{k l}=\Delta \phi_{k}-\Delta \phi_{l}=\Delta f_{k}\left(\Delta f_{1}\right)+\Delta f_{k}\left(\Delta f_{n e l+1}\right)-\Delta f_{l}\left(\Delta f_{1}\right)-\Delta f_{l}\left(\Delta f_{n e l+1}\right)
$$

\section{Application to the TEP and MIRAS instruments}

In this section we study the performance and requirements of the application of the RSC method to two particular instruments: TEP, a volume imaging atmospheric radar, and MIRAS, an L-band synthetic aperture radiometer under construction.

\subsection{TEP}

TEP (Mead et al. 1998) is an ultrahigh frequency (UHF) digital-beamforming phased-array designed to provide fine-scale imagery of clear-air scattering in the atmospheric boundary layer. A transmit horn illuminates a $25^{\circ}$ volume and the scattered signal is received by each of the 90 elements of a hexagonal array (figure 5(a)); and a beamforming algorithm is implemented to combine information from all elements, focusing 40 adjacent $3.5^{\circ}$ synthetic beams with a range resolution of $30 \mathrm{~m}$ (figure $5(b)$ ). TEP provides four-dimensional [three-dimensional (3D) volume and time] views of the atmospheric turbulence structure (the structure constant $C_{n}^{2}$ is related to the volumetric radar reflectivity at each range and focused pixel $\eta_{\mathrm{VOL}}=0.38 C_{n}^{2} \lambda^{-1 / 3}$ ) and $3 \mathrm{D}$ wind fields within a volume from $200-1500 \mathrm{~m}$. The basic parameters of TEP are listed in table 3 . In addition to the injection of a CW (continuous wave) tone, TEP can be calibrated by RSC using the shortest available range gates to maximize the SNR (Attia and Steinberg 1989, Hopcraft 1997), which are limited to $r \approx 220 \mathrm{~m}$ due to ground clutter. The phase statistics of partially correlated random variables have been studied in interferometric Synthetic 


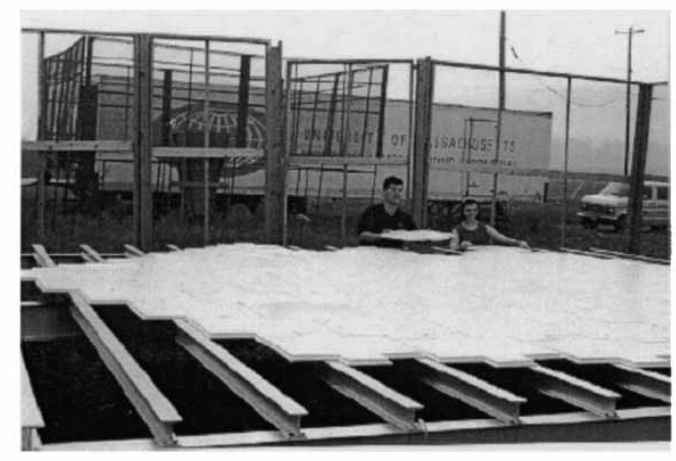

(a) (b)

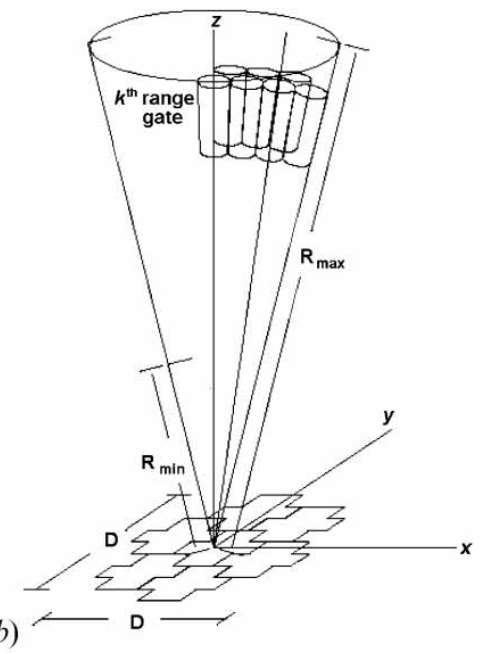

Figure 5. (a) TEP 90-element hexagonal array in the field. (b) A transmit horn illuminates a $25^{\circ}$ volume and the scattered signal is received by each of the 90 elements of a hexagonal array; and a beamforming algorithm is implemented to combine information from all elements, focusing 40 adjacent $3.5^{\circ}$ synthetic beams with a range resolution of $30 \mathrm{~m}$.

Aperture Radar (SAR) (Carande 1994):

$$
\sigma_{\phi}=\frac{1}{\sqrt{2 N}} \frac{\sqrt{1-\left|\hat{V}_{k l}\right|^{2}}}{\left|\hat{V}_{k l}\right|}
$$

where $\left|\hat{V}_{k l}\right|=\left|V_{k l}\right| /\left(1+\mathrm{SNR}^{-1}\right), \quad N=\tau_{\text {int }} f_{s}$ is the number of samples being averaged and SNR is the signal-to-noise ratio at receivers' output, $\tau_{\text {int }}$ is the integration time and $f_{s}$ is the sampling frequency (twice the signal bandwidth). The number of samples $N$ required to achieve a specific value of $\sigma_{\phi}$ can be determined by computing the SNR as a function of the radar reflectivity at $r=220 \mathrm{~m}$ and TEP parameters (table 3 ). Long integration times are limited by system drifts, mainly due to thermal variations. The integration time for calibration is then limited to a fraction of the time of these fluctuations, which depends on the particular conditions of operation.

Figure 6 shows the computed number of samples $N$ required to achieve a given phase error during calibration (equation (13)) as a function of the structure constant $\left(C_{n}^{2}\right)$ to be measured. For example, assuming $\tau_{\text {int }}=10 \mathrm{~s}$ and echoes from a single range gate, phase calibration for a calm and dry atmosphere $\left(C_{n}^{2} \approx 5 \cdot 10^{-16}\right)$ can be achieved with a phase error $\sigma_{\phi} \approx 31^{\circ}(\mathrm{SNR} \approx-6.5 \mathrm{~dB}$ at $220 \mathrm{~m})$, or for a warm, humid and turbulent atmosphere $\left(C_{n}^{2} \approx 10^{-12}\right)$ with $\sigma_{\phi} \approx 5.4^{\circ}(\mathrm{SNR} \approx 26.5 \mathrm{~dB}$ at $220 \mathrm{~m}$ ). For a given SNR, amplitude calibration is much more sensitive to random errors due to the error amplification $\left(g_{k}=\exp \left(G_{k}\right)\right)$. Amplitude calibration is then performed taking into account that the power received by the in-phase and quadrature channels of all elements must be the same.

As an indicator of performance, figure 7 shows a comparison between subsequent RSC-derived phase calibrations for the TEP array (Lopez-Dekker et al. 2002). For visualization purposes, we show the Fourier transform of the quotient of two 
Table 3. Basic parameters of the Turbulent Eddy Profiler.

Parameter

Frequency

Peak power

$915 \mathrm{MHz}$

Pulse width

$25 \mathrm{~kW}$

Pulse repetition frequency

$200 \mathrm{~ns}$

Transmitter antenna beamwidth (corrugated horn)

$40 \mathrm{KHz}$

$25^{\circ}$

Transmitter antenna/line efficiency

Receiving antenna: hexagonal array

0.9

Elementary receiver antenna beamwidth

91 elements

(seven-microstrip patch array)

Minimum separation between antenna phase centres

$32^{\circ}$

(wavelengths)

Receiver antenna/line efficiency

1.65

System temperature

0.8

Receiver bandwidth

$\sim 175 \mathrm{~K}$

$2.5 \mathrm{MHz}$

Quantization

10 bits

Correlation amplitude at the shortest baseline (1.65 wavelengths)

$\left|V_{k l}\right|=0.18$ (determined from transmitter antenna (Hopcraft 1997))

calibration vectors. In the absence of errors, this would be unity and the resultant 'beam pattern' would reflect that of an ideal aperture (no amplitude or phase errors). Figure 7(a) shows such an idealized result for a 61-element hexagonal lattice with a few missing elements. Figure 7(b) shows the quotient of two RSC-derived phased calibrations from the same array, each derived from approximately $10^{5}$ independent data samples of clear-air scatter of mean $C_{n}^{2}$ between $10^{-14}$ and $10^{-13}$.

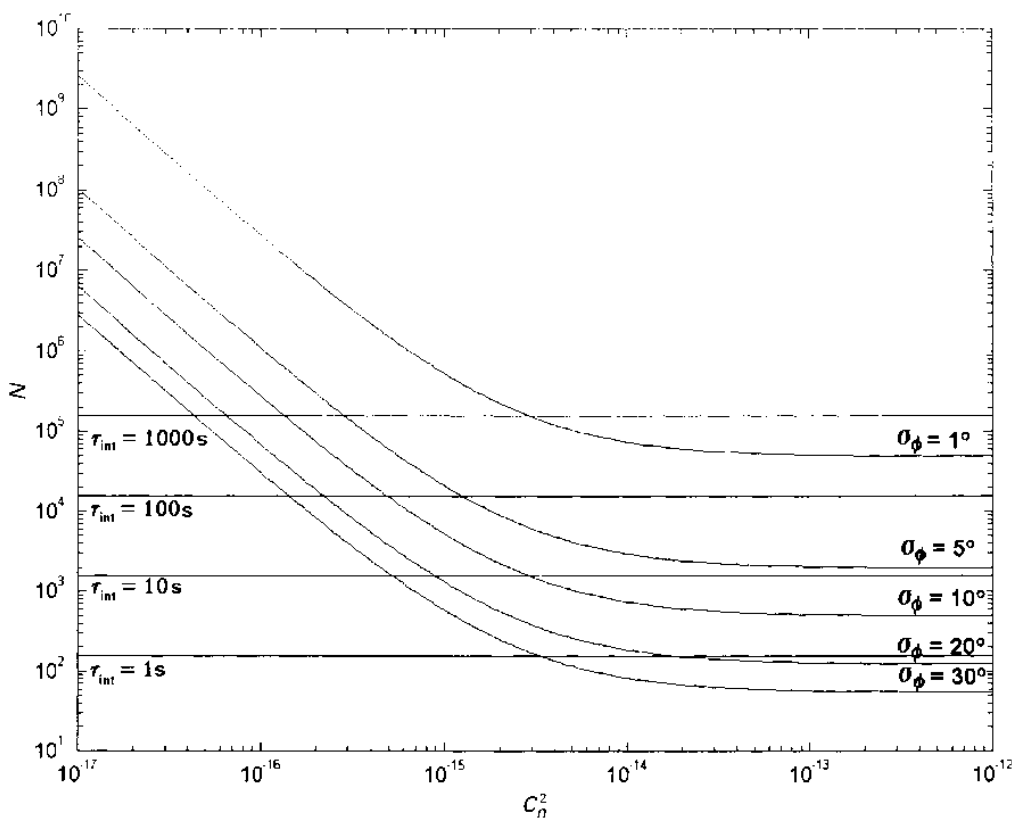

Figure 6. TEP: number of samples required to achieve a given $\sigma_{\phi}$ during calibration vs structure constant $C_{n}^{2}$. Radar reflectivity $\eta_{\mathrm{VOL}}=0.38 C_{n}^{2} \lambda^{-1 / 3}$. 


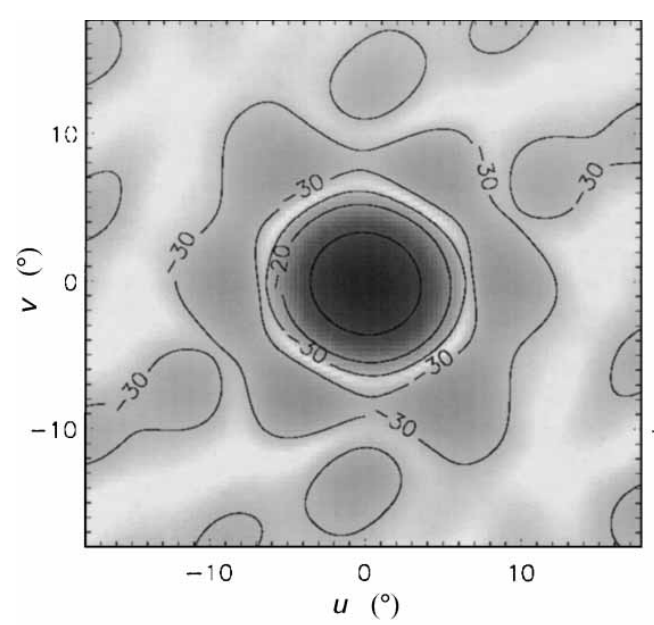

(a)

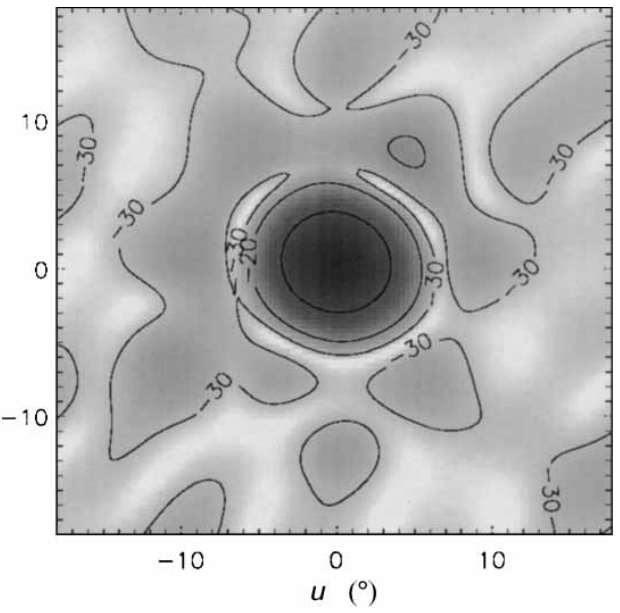

(b)

Figure 7. Comparison between subsequent RSC-derived phase calibrations for the TEP array: (a) idealized result for a 61-element hexagonal lattice with a few missing elements; $(b)$ quotient of two RSC-derived phased calibrations from the same array, derived from $\sim 10^{5}$ independent data samples of clear-air scatter $\left(C_{n}^{2}=10^{-13}-10^{-14}\right)$.

In figure 7(b), the location of the beam maximum indicates the change in pointing between calibrations (which is quite small), while the sidelobe level indicates the relative impact of random phase and amplitude estimation errors. In both cases, a taper has been applied to reveal more clearly the limit on sidelobe performance imposed by amplitude and phase errors. This visualization enables separation of pointing error that induces linear-phase variation across the aperture and random error that contributes to the sidelobes. It does not, however, show actual pointing and actual sidelobe behaviour of the array, which depend upon other factors such as variations in individual antenna element patterns. In any case, we note only a small degradation in the pattern between cases $a$ and $b$.

\subsection{MIRAS}

MIRAS is a dual polarization L-band Y-shaped interferometric radiometer (figure 8) for the measurement of soil moisture and sea salinity (Martin-Neira and Goutoule 1997, Silvestrin et al. 2001). In this instrument, brightness temperature maps are formed by a Fourier synthesis process $[I(\xi, \eta) \leftrightarrow V(u, v)$; equation(1)]. In its original configuration it had 43 antennas per arm spaced 0.89 wavelengths. In order to demonstrate the concept and make it suitable for a small platform, a scaled-down version with 23 antennas per arm was proposed as the Soil Moisture and Ocean Salinity Mission (SMOS) Earth Explorer Opportunity Mission to the European Space Agency (ESA). Its structure is depicted in figure 3(a) and its basic parameters are listed in table 4.

Because of the high radiometric accuracy required, internal calibration by correlated noise injection is foreseen (Torres et al. 1996). Redundant space calibration can then be applied to correct antenna pattern constant phase errors and amplitude errors induced by antenna losses, since noise is injected right after the antenna. The amplitude term $g_{0}$ has to be accurately characterized on the ground. 


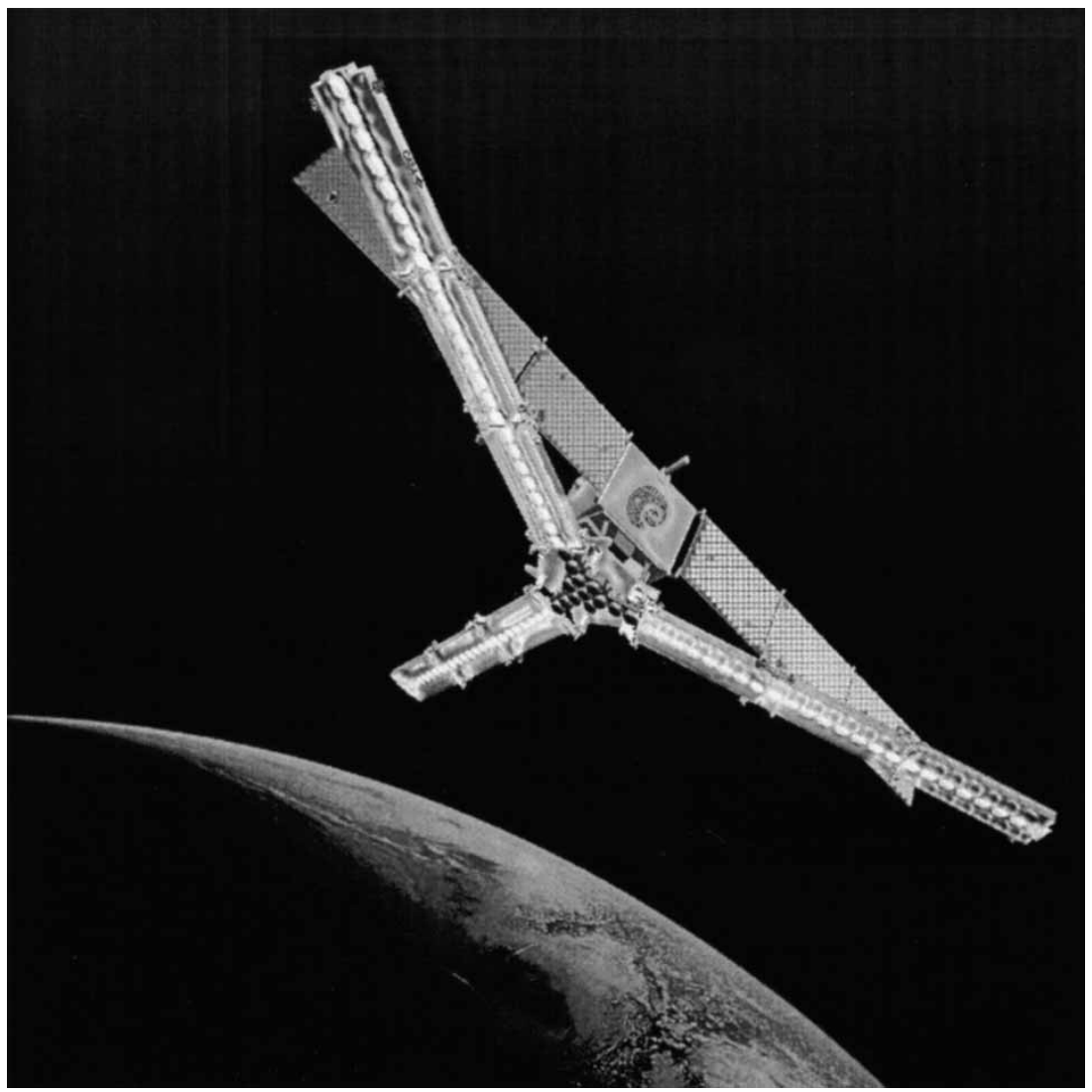

Figure 8. Artist's view of MIRAS aboard the SMOS Earth Explorer Opportunity Mission (courtesy of EADS-CASA, Space Division, Spain, prime contractor of the MIRAS instrument).

The rms phase and amplitude errors can be calculated from the parameters listed in table 4 and equation (9) applied to the Y-structure, leading to $\sigma_{\phi}=0.4^{\circ}-1.3^{\circ}$ and $\sigma_{g}=0.008-0.023$. In order to reduce the uncertainty of the phase/amplitude estimates, $N$ measurements must be averaged ${ }^{2}$. In the worst cases $\left(\sigma_{\phi}=1.3^{\circ}\right.$ and $\left.\sigma_{g}=0.023\right)$, the $N$ is 7 for the phase, and 132 for the amplitude ${ }^{3}$.

Note also that the application of phase and amplitude closure relationships would lead to an overdetermined system of equations, usually involving longer baselines and exhibiting poorer performance due to lower SNR.

\section{Conclusions}

This paper has reviewed the principles of phase and amplitude redundant space calibration methods, pointing out that they are common to beamforming radars

\footnotetext{
${ }^{2}$ Correlations cannot be averaged due to the changes in the scene caused by platform movement $\left(\sim 7.5 \mathrm{~km} \mathrm{~s}^{-1}\right)$, so phase/amplitude estimates must be averaged.

${ }^{3} N=132$ is close to $N=100$ measurements $\left(\tau_{\text {int }}=0.3 \mathrm{~s}\right)$ required by the noise injection calibration (Torres et al. 1996).
} 
Table 4. Basic parameters of the Microwave Imaging Radiometer by Aperture Synthesis.

Parameter

Frequency

Bandwidth

Number of elements per arm

Antenna beamwidth

Minimum separation between antenna

phase centres (wavelengths)

Receivers' noise temperature

System temperature

Integration time $\left(\tau_{\text {int }}\right)$

1 bit/2 level digital correlator: $\tau_{\text {effective }} / \tau$

Platform speed

Standard deviation of the individual visibility samples (Camps et al. 1998)

Standard deviation of the individual visibility samples (Camps et al. 1998)

Correlation amplitude at the shortest baseline $(0.89$ wavelengths) [determined from the $T_{B}$ scene (Camps et al. 1997b)]

Phase and amplitude errors for $1 \mathrm{~K}$ radiometric accuracy (Camps et al. 1997a)

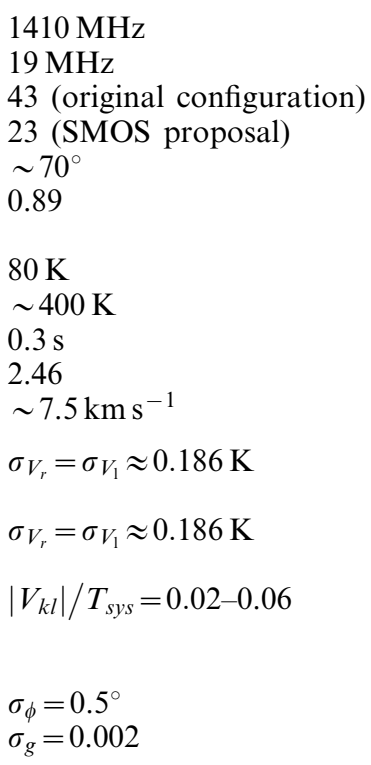

and interferometric radiometers. The RSC method has been applied to hexagonal and $\mathrm{Y}$-shaped arrays, showing that in both configurations:

(a) regardless of the number of equations used, the rank of the system of equations is at least two less than the number of phase unknowns. These two degrees of freedom represent a pointing error of the focused or synthetic beam. To correct it, two instrumental phases need to be determined;

(b) except for the configuration named as case 1, elements' amplitude can be determined without ambiguity, except for a normalization constant $g_{0}=1$. However, the system of equations is very sensitive to instrumental errors and it is better to use the fact that the power of the in-phase and quadrature channels is the same;

(c) system performance relies on the accuracy of the phase references (e.g. $f_{1}$ and $f_{2}$ or $\phi_{01}$ and $\phi_{02}$ ), since errors in their estimation propagate and are amplified to other elements in the array;

(d) errors not taken into account by the $\mathrm{RSC}^{4}$ method must be kept low enough by careful hardware implementation and/or corrected for by other means to guarantee RSC performance;

(e) the shortest redundant baselines must be used to implement the RSC method in order to have the largest SNR;

(f) in an interferometric radiometer, RSC does not require any special additional hardware, since the basic products are already complex cross-correlations. RSC is complementary to any other existing calibration methods.

Finally, the application of RSC to two particular instruments has been discussed. In TEP, the technique has already been successfully implemented. In

\footnotetext{
${ }^{4}$ These are second order phase/amplitude errors associated to the particular pair of elements forming the baseline.
} 
MIRAS, it is proposed as a combined method: noise injection for receiver errors, and RSC for constant antenna errors. Antenna voltage pattern direction dependent errors can only be accounted for during the image reconstruction process.

\section{Acknowledgments}

This work has been partially supported by the US Army Research Office (Atmospheric Sciences) and the Vertical Transport and Mixing Program of the US Department of Energy Office of Biological and Environmental Research (SJF and PL-D) and the Spanish Ministry of Culture (PR 98 46131105, sabbatical leave fellowship of AC at the Microwave Remote Sensing Laboratory, University of Massachusetts, during 1999).

\section{References}

Attia, E. H., and Steinberg, B. D., 1989, Self-cohering large antenna arrays using the spatial correlation properties of radar clutter. IEEE Transactions on Antennas and Propagation, 37, 30-38.

Bara, J., Camps, A., Torres, F., and Corbella, I., 1998, Angular resolution of twodimensional hexagonally sampled interferometric radiometer. Radio Science, 33, 1459-1473.

Born, M., and Wolf, E., 1975, Principles of Optics, 5th edn (Oxford: Pergamon Press).

Camps, A., BarÁ, J., Torres, F., Corbella, I., and Romeu, J., 1997a, Impact of antenna errors on the radiometric accuracy of large aperture synthesis radiometers. Study applied to MIRAS. Radio Science, 32, 657-668.

Camps, A., Torres, F., Bará, J., Corbella, I., Pino, M., and Martín Neira, M., 1997b, Evaluation of MIRAS spaceborne instrument performance: snap shot radiometric accuracy and its improvement by means of pixel averaging. The European Symposium on Aerospace Remote Sensing, Conference on Sensors, Systems and Next Generation Satellites III, London, UK, September 1997, Proceedings of the SPIE, 3498, 43-52.

Camps, A., Bará, J., Torres, F., Corbella, I., and Monzón, F., 1998, Experimental validation of radiometric sensitivity in correlation radiometers. Electronics Letters, 34, 2377-2378.

CARANDE, R. E., 1994, Estimating ocean coherence time using dual-baseline synthetic aperture radar. IEEE Transactions on Geoscience and Remote Sensing, 32, 846-854.

Hamaker, J. P., O'sullivan, J. D., and Noordam, J. E., 1977, Image sharpness, Fourier optics and redundant-spacing interferometry. Journal of the Optical Society of America, 67, 1122-1123.

HopCrAfT, G., 1997, The Turbulent Eddy Profiler: a digital beam-forming system for clear air turbulence measurement. PhD dissertation, University of Massachusetts, Amherst, USA.

Lopez-Dekker, P., Farquharson, G., and Frasier, S. J., 2002, entropy based phase calibration of antenna arrays for digital beamforming remote sensing radars. Proceedings of the 2002 IEEE Radar Conference, Long Beach, CA, USA, 22-25 April 2002 (Piscataway, NJ: IEEE).

MARtín-Neira, M., and Goutoule, J. M., 1997, MIRAS - a two-dimensional aperture-synthesis radiometer for soil-moisture and ocean salinity observations. ESA Bulletin, 92, 95-104.

Mead, J. B., Hopcraft, G., Frasier, S. J., Pollard, B. D., Cherry, C. D., Schaubert, D. H., and McIntosh, R. E., 1998, A volume-imaging radar wind profiler for atmospheric boundary layer turbulence studies. Journal of Atmospheric and Oceanic Technology, 15, 849-859.

Sivestrin, P., Berger, M., Kerr, Y., and Font, J., 2001, ESA's Second Earth Explorer Opportunity Mission: The Soil Moisture and Ocean Salinity Mission-SMOS. IEEE Geoscience and Remote Sensing Newsletter, March, No.118, pp.11-14.

Thompson, R., Moran, J. M., and Swenson, G. W., 1986, Interferometry and Synthesis in Radio Astronomy (New York: John Wiley).

Torres, F., CAmps, A., BARÁ, J., Corbella, I., and Ferrero, R., 1996, On-board phase and modulus calibration of large aperture synthesis radiometers: study applied to MIRAS. IEEE Transactions on Geoscience and Remote Sensing, 34, 1000-1009. 